\title{
Single-Frequency Ytterbium-Doped Fibre Laser Stabilized by Spatial Hole Burning
}

\author{
R. Paschotta, J. Nilsson, L. Reekie, A. C. Tropper, and D. C. Hanna \\ Optoelectronics Research Centre, Southampton SO17 1BJ, England \\ submitted to Optics Letters 29108136
}

\begin{abstract}
We have exploited spatial hole burning to achieve remarkably stable singlefrequency operation and mode-hop-free tuning over 300 free spectral ranges in an ytterbium-doped fibre laser with a simple standing-wave geometry. This approach makes possible stable and narrow-linewidth single-frequency fibre lasers which do not require components such as Faraday isolators, fibre couplers, or Fabry-Perot filters.
\end{abstract}

PACS: $42.55 . \mathrm{W}, 42.60 . \mathrm{P}$

Fibre lasers in a simple standing-wave geometry can not usually generate a singlefrequency (SF) output because of the well-known effect of "spatial hole burning". (SHB) in the gain medium. This problem is usually solved by using either a unidirectional ring cavity $^{1}$ (in which SHB does not occur) or a very short (few $\mathrm{cm}$ long) standing-wave cavity combined with one or two narrow-band Bragg fibre gratings ${ }^{2}$ which select a single longitudinal mode despite the presence of SHB). However, the latter approach works only with very short cavities, thus often limiting the pump efficiency and leading to a large linewidth compared to long ring lasers. On the other hand, unidirectional ring lasers need expensive components such as Faraday isolators and fibre couplers and still have a tendency for mode-hopping which may need to be eliminated with an additional narrow-band optical filter ${ }^{1}$.

It has been noted that for a three-level laser transition, SHB in a doped but unpumped, thus reabsorbing section within the laser cavity has the beneficial effect of stabilizing SF operation based on the principle that the lasing mode partially saturates the absorption and suffers a lower loss than all competing modes. This effect has been used for linewidth narrowing of an erbium-doped fibre laser ${ }^{3}$, although stable SF operation was not achieved in that case. The laser cavity contained two sections of erbium-doped fibre, one section being unpumped and therefore reabsorbing. The authors tried to eliminate SHB in the pumped section by selecting counterpropagating orthogonal polarization states in this region. The reason why stable SF operation was not achieved is not identified in the paper; possibly the fibre birefringence prevented strict orthogonality of polarization states over the whole $50 \mathrm{~cm}$ length of amplifying fibre. We have found that the birefringent beat-length of rare-earthdoped fibres is typically well below $1 \mathrm{~m}$ so that it is difficult to eliminate SHB by polarization control. 
A different approach was demonstrated in Ref. 4: an unpumped section of erbium-doped fibre was incorporated into a ring laser in which the pumped fibre amplified a travelling wave which does not lead to SHB. Even without the reabsorbing section, the laser was already operating on a single frequency, but with frequent mode-hops. With the reabsorbing section, the rate of mode-hopping was significantly reduced (to one mode-hop every few minutes).

In this letter we report that under certain circumstances the stabilizing effect of SHB in an unpumped section of a standing-wave laser cavity can override the destabilizing effect of SHB in the amplifying section, even if SHB is not reduced (e.g. by using orthogonal polarization states) in the amplifying section. The magnitude of pump and signal crosssections is of crucial importance for this mechanism to work. A large pump absorption cross-section is needed for two reasons. Firstly, it causes the pump power to be absorbed within a short length of fibre so that we obtain a pumped and an unpumped section of fibre without the need to remove the residual pump light at some point e.g. with a WDM coupler. The second and more important reason is that the large pump cross-sections (both for absorption and emission) reduce the effect of SHB in the pumped section. To see this we consider the steady-state fraction $n_{2}$ of excited ions, given by

$n_{2}=\frac{R_{12}+W_{12}}{R_{12}+R_{21}+W_{12}+W_{21}+A_{21}}$

where $R_{12}$ and $R_{21}$ are the transition rates per ion induced by the pump, $W_{12}$ and $W_{21}$ the rates caused by the signal wave, and $A_{21}$ the spontaneous emission rate. The transition rates depend on intensities and cross-sections, e.g. $R_{12}=\sigma_{12}^{(p)} I_{\mathrm{p}} / h \nu_{\mathrm{p}}$ where $I_{\mathrm{p}}$ is the pump intensity (in $\mathrm{W} / \mathrm{m}^{2}$ ) and $\sigma_{12}^{(p)}$ the pump absorption cross-section. From these equations it is clear that the spatial modulation of the inversion density caused by a SF signal wave is smaller where the pump rates $R_{12}$ and $R_{21}$ are large, especially when the signal rates $W_{12}$ and $W_{21}$ are small; therefore the destabilizing effect of SHB in the pumped section can be significantly weaker than the stabilizing effect in the absorbing region even if the total reabsorption loss is smaller than the total gain in the pumped section (as required in order to reach threshold).

According to these considerations we looked for a laser transitions with large pump crosssections and smaller signal cross-sections. We found that a laser made of Yb-doped silica fibre ${ }^{5}$ can meet these conditions if it is pumped at $975 \mathrm{~nm}$ and operating e.g. at $1040 \mathrm{~nm}$ : then we have $\sigma_{12}^{(p)}=\sigma_{21}^{(p)}=2600 \cdot 10^{-27} \mathrm{~m}^{2}, \sigma_{21}^{(p)}=513 \cdot 10^{-27} \mathrm{~m}^{2}$, and $\sigma_{12}^{(p)}=25 \cdot 10^{-27} \mathrm{~m}^{2}$ (see Ref. 5 for typcial spectra).

To test this new approach, we used a $6 \mathrm{~m}$ long germanosilicate fibre with a $930 \mathrm{~nm}$ cut-off, a numerical aperture of 0.21 and $2300 \mathrm{ppm}$ (by weight) Yb doping. We calculated with a numerical computer model that in this case the pump power of $\sim 20 \mathrm{~mW}$ (launched) is absorbed within $\sim 0.5 \mathrm{~m}$; therefore the other $5.5 \mathrm{~m}$ of fibre act as the absorber. The cavity was formed by a dielectric mirror butt-coupled to the pumping end (with $80 \%$ transmittance for the $975 \mathrm{~nm}$ pump and $99 \%$ reflectivity at $1040 \mathrm{~nm}$ ) and a fibre grating (with $92 \%$ reflection at $1040 \mathrm{~nm}$, bandwidth $\sim 0.1 \mathrm{~nm}$, in a short piece of undoped fibre) spliced to the other end. A frequency-selective element such as the fibre grating is necessary in order to prevent lasing at longer wavelengths where the reabsorption is weak. Fig. 2 shows the calculated power distributions for a typical operating point. 
The output bearn at $1040 \mathrm{~nm}$ was collimated with a microscope objective and analyzed with a Fabry-Perot interferometer ( $500 \mathrm{MHz}$ free spectral range). The incident pump power (from a Ti:sapphire laser) at threshold was $\sim 70 \mathrm{~mW}$. Some bistability (as predicted in Ref. 6 ) was observed: once lasing had started, the pump power had to be reduced by a few milliwatts in order to stop lasing. For a pump power of $80 \mathrm{~mW}$, lasing occurred either on a single mode or on several modes simultaneously with a relatively stable pattern of intensities. Singlemode operation could be achieved by a slight bending of a part of the fibre (thus influencing the polarization states in the fibre). The bent fibre was simply held on the table with tape. For pump powers only slightly above threshold (e.g. $72 \mathrm{~mW}$ ), we observed occasional modehops. For somewhat higher pump powers $(\sim 75 \ldots 95 \mathrm{~mW}), \mathrm{SF}$ operation was very stable without mode-hopping (over observation times of at least several minutes), except when the laser was deliberately and grossly disturbed e.g. by knocking on the optical table. After a short interruption of the pump beam, lasing usually resumed on a different (but single) mode. For higher pump powers around $90 \mathrm{~mW} \ldots 100 \mathrm{~mW}$, the laser tended to start lasing on two-modes when the optical table was knocked, especially when the polarization adjustment was not correct; SF operation could then be restored only by briefly interrupting the pump beam.

For still higher pump powers $(\sim 105 \ldots 130 \mathrm{~mW})$, the laser entered a noisy multi-mode regime. The power would now be distributed over at least $\sim 50$ or perhaps even hundreds of modes. The power per mode was much lower now and fluctuated quickly between the modes, as is typical for standing-wave cavities without a reabsorbing region. When the pump wavelength was detuned slightly from the $975 \mathrm{~nm}$ absorption peak, noisy multifrequency operation was observed even just above threshold. This demonstrated that the large pump cross-sections at $975 \mathrm{~nm}$ are essential for stable SF operation.

For pump powers in the middle of the stability region (e.g. $85 \mathrm{~mW}$ at $975 \mathrm{~nm}$ ), the laser could be tuned without mode-hops by simply heating a part of the fibre (other than the fibre grating) with a $60 \mathrm{~W}$ light bulb. We could in this way tune the laser by up to $5 \mathrm{GHz}(300$ free spectral ranges) before a mode-hop occured. This tuning range is a significant fraction of the $30 \mathrm{GHz}$ bandwidth of the fibre grating. We believe that the tuning range is indeed limited only by the bandwidth of the grating.

When only the fibre grating was heated, mode-hops occurred after a very small frequency change. Before a mode-hop occurred, the output power would significantly increase, often by more than a factor of 5 in some earlier experiments in which we used a grating with $97 \%$ reflectivity. This is because heating the grating moves its reflection maximum to longer wavelengths while the cavity length and thus the frequency of the lasing mode are not significantly changed. Consequently the reduced reflectivity at the lasing wavelength leads to more efficient output coupling and thus an increased output power. We calculated with the computer model that a power enhancement by a factor of 5 corresponds to a decrease of the grating reflectivity from $97 \%$ to $\sim 75 \%$. From this we conclude that SHB generated a gain advantage of at least $1 \mathrm{~dB}$ for the lasing mode compared to any competing modes, which explains the high stability of the SF operation.

The maximum SF output power (with the $92 \%$ grating) was $0.5 \mathrm{~mW}$; the slope efficiency was $\sim 1 \%$ in the single-mode regime. In the multimode regime the output power grew faster than linearly with input power (see Fig. 1). We believe the reason to be that at higher powers the signal bandwidth increases so that a part of the lasing power is contained in 
modes which experience a lower grating reflectivity which enhances the output coupling efficiency.

A somewhat higher slope efficiency and output power should be achievable with a grating of lower reflectivity, while the intracavity signal power could not be increased significantly because the excited state population in the absorber section would be saturated even near the nodes of the signal wave, thus reducing the stabilizing SHB effect in the absorber. Another problem with significantly larger signal powers in a long fibre laser is that enough stimulated Brillouin gain could be generated to excite some other mode, although the narrow bandwidth of the grating reflector provides some discrimination against such a mode which would have a lower frequency by $\sim 17 \mathrm{GHz}$.

We have tried several other lengths of fibre and found stable SF operation over a wide range between about $1.5 \mathrm{~m}$ and $10 \mathrm{~m}$. For a short fibre length (e.g. $2 \mathrm{~m}$ ), the stability was poor because the unpumped reabsorbing section was short. For longer fibre lengths we observed significant broad-band ASE (amplified stimulated emission), centred at $\sim 1030 \mathrm{~nm}$; for example, the $6 \mathrm{~m}$ long laser produced $\sim 50 . .100 \mu \mathrm{W}$ of ASE at the output just below threshold.

Due to the long length of our laser cavity we expect the linewidth to be very narrow, probably between $1 \mathrm{kHz}$ and $10 \mathrm{kHz}$ as is usually obtained with long fibre ring lasers ${ }^{7,8}$ but not with short standing-wave cavities (see e.g. Ref. 9). We have not measured the linewidth of our laser. The usual technique of using a long self-heterodyne delay line does not give sufficient resolution since the length of the delay line is restricted by the fibre loss of $\sim 1 \mathrm{~dB} / \mathrm{km}$ at $1040 \mathrm{~nm}$. It may prove necessary to build two similar lasers in order to measure the spectral width of the beat signal.

We expect that the method described here can be applied to other laser transitions as well. For erbium-doped fibre lasers with the usual pump and signal wavelengths of $980 \mathrm{~nm}$ and $1530 \ldots 1570 \mathrm{~nm}$, respectively, the relative magnitude of cross-sections are unfortunately not suitable: the pump absorption cross-section is usually no larger than the signal crosssections. However, the scheme might work with a green pump beam or at longer signal wavelengths where the cross-sections are smaller.

In conclusion, we have presented a new approach to obtaining single-frequency operation from fibre lasers. Remarkable stability and tuning over 300 free spectra ranges without mode-hopping have been achieved in an $\mathrm{Yb}$-doped laser with a very simple standing-wave configuration, not involving expensive components like Faraday isolators, fibre couplers, or narrow-band optical filters. As our approach allows the use of very long laser cavities, a very narrow linewidth should be achievable. The appropriate relative magnitudes of pump and signal cross-sections, as provided by the Yb-doped fibre, appear to be of crucial importance.

\section{ACKNOWLEDGMENTS}

We thank W. H. Loh for useful discussions. 


\section{REFERENCES}

1. J. L. Zyskind, J. W. Sulhoff, Y. Sun, J. Stone, L. W. Stulz, G. T. Harvey, D. J. DiGiovanni, H. M. Presby, A. Piccirilli, U. Koren, and R. M. Jopson, Electron. Lett. 27, 2148 (1991).

2. G. A. Ball and W. H. Glenn, J. Lightwave Technol. 10, 1338 (1992).

3. M. Horowitz, R. Daisy, B. Fischer, and J. Zyskind, Electron. Lett. 30, 648 (1994).

4. Y. Cheng, J. T. Kringlebotn, W. H. Loh, R. I. Laming, and D. N. Payne, Opt. Lett. 20, 875 (1995).

5. H. M. Pask, R. J. Carman, D. C. Hanna, A. C. Tropper, C. J. Mackechnie, P. R. Barber, and J. M. Dawes, IEEE J. Sel. Topics in Quantum Electron. 1, 2 (1995).

6. M. Horowitz, R. Daisy, B. Fischer, and J. L. Zyskind, Opt. Lett. 19, 1406 (1994).

7. K. Iwatsuki, H. Okamura, and M. Saruwatari, Electron. Lett. 26, 2033 (1990).

8. G. J. Cowle, D. N. Payne, and D. Reid, Electron. Lett. 27, 229 (1991).

9. J. T. Kringlebotn, J.-L. Archambault, L. Reekie, and D. N. Payne, Opt. Lett. 19, 2101 (1994). 


\section{FIGURES}

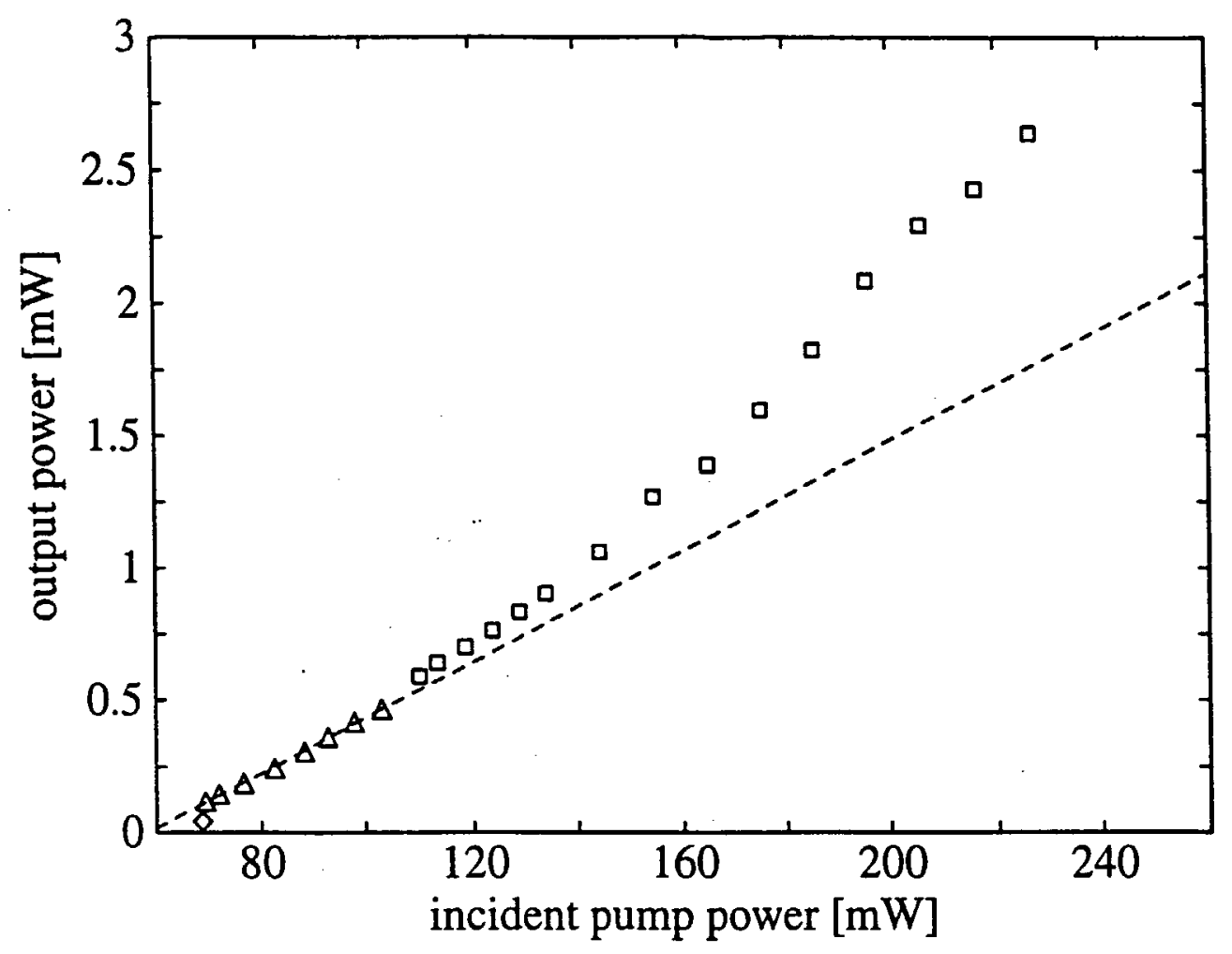

Fig: 1. $\quad 1040 \mathrm{~nm}$ output power versus incident $975 \mathrm{~nm}$ input power for a $6 \mathrm{~m}$ long laser as described in the text. Triangles: single-frequency operation. Dashed line: linear fit for points with single-frequency operation. Squares: noisy multi-mode operation. Rhomb: ASE power just below threshold. 


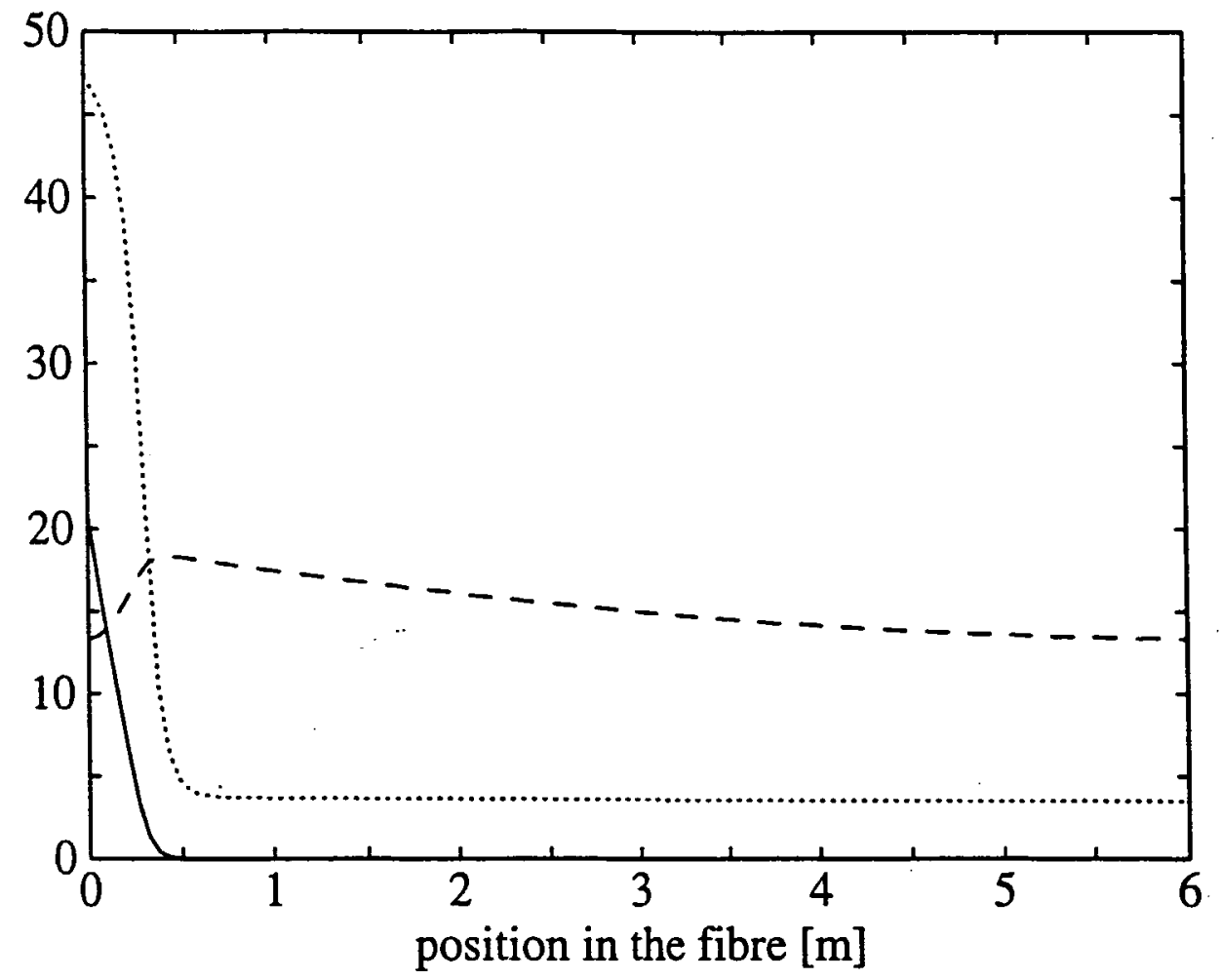

Fig. 2. Power distribution along the fibre in our Yb-doped fibre laser, calculated for $100 \mathrm{~mW}$ of incident pump power (threshold: $70 \mathrm{~mW}$ ). Solid curve: pump power in $\mathrm{mW}$. Dashed curve: signal power in $\mathrm{mW}$ (sum of forward and backward propagating waves). Dotted curve: excited state population in percent. Note that in the region without pump power the excited state population is held near its saturation value by the signal wave. 